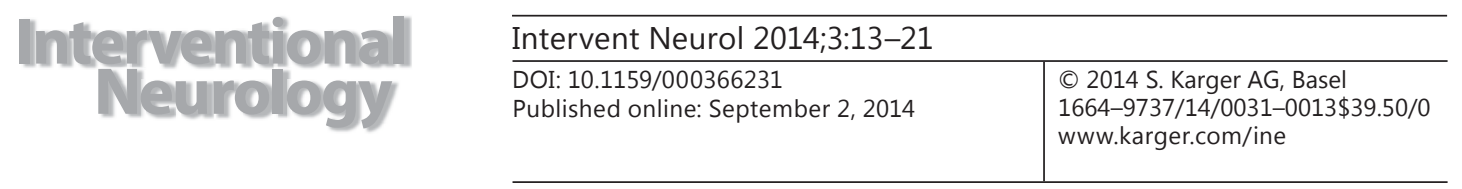

\title{
Review
}

\section{Hemodynamic Changes and Baroreflex Sensitivity Associated with Carotid Endarterectomy and Carotid Artery Stenting}

\author{
Qinqin Cao Jun Zhang Gelin Xu \\ Department of Neurology, Jinling Hospital, Nanjing University School of Medicine, \\ Nanjing, China
}

\section{Key Words}

Hemodynamic changes - Baroreflex sensitivity - Carotid endarterectomy ·

Carotid artery stenting

\begin{abstract}
Atherosclerotic carotid lesion is a major cause of stroke which accounts for up to $20 \%$ of ischemic stroke. Aggressive treatment of carotid stenosis may prevent stroke. Currently, carotid endarterectomy (CEA) and carotid artery stenting (CAS) are the first-line treatments for severe carotid stenosis. CEA is superior to medical therapy in preventing stroke and cardiovascular death. CAS has emerged as an alternative to CEA in recent years due to its less invasive nature. However, both CEA and CAS may be associated with adverse hemodynamic changes as well as a variation of carotid baroreflex sensitivity. There is no consensus on which of these two methods is more advantageous concerning the procedure-related hemodynamic changes. This article reviews the hemodynamic changes and baroreflex sensitivity after CEA and CAS.
\end{abstract}

(C) 2014 S. Karger AG, Basel

\section{Introduction}

Carotid endarterectomy (CEA) and carotid artery stenting (CAS) are the fist-line treatments for severe atherosclerotic carotid stenosis. However, procedure-related hemodynamic changes and variation of carotid baroreflex sensitivity are very common and become a major 
disadvantage of these treatments. These adverse effects may prolong hospitalization and increase procedure-related morbidity and mortality $[1,2]$. This review summarizes the hemodynamic changes after CEA and CAS. The possible mechanisms and prevention strategies are also discussed.

\section{Carotid Sinus Baroreflex}

Carotid sinus baroreceptors (CSBs) are located in the carotid sinus, at the bifurcation of the common carotid artery. CSBs are stretch receptors monitoring the changes of blood pressure (BP) through the distension of the vessel wall. They are innervated by branches of the IX and X cranial nerves. Increased BP activates the feedback circuit of the carotid baroreflex which involves several medullary nuclei, such as the nucleus tractus solitarii (NTS), the caudal ventrolateral medulla (CVLM) and the rostral ventrolateral medulla (RVLM) $[3,4]$.

The NTS is the first station to receive the nerve impulses and perhaps one third of these neurons may be involved in the baroreflex [5]. Baroreceptor afferent fibers terminate mainly in the intermediate portion of the NTS, but there are differences between different types of fibers (myelinated, A-fiber and unmyelinated, C-fiber). The C-fiber may tend to be located more often medially in the NTS while the A-fiber more laterally. The discharge characteristics of the two types of fibers are also different. The A-fiber in rats has a lower threshold and a higher frequency than the C-fiber at the same pressure. Also, they provide different types of information to the central neurons [6]. The A-fiber may be involved in rapid adjustment of systemic BP, whereas the C-fiber mainly maintains the baseline level [7]. The afferent fibers contain and release glutamate [8] which can excite the second-order neurons in the NTS. These NTS neurons in turn send excitatory signals to neurons in the CVLM. CVLM neurons are interneurons in the baroreflex [9] and receive direct synaptic contacts from NTS efferent terminals [10]. These neurons are GABAergic [11] and project directly to bulbospinal neurons in the RVLM [10]. Baroreceptor neurons of the CVLM inhibit sympathetic neurons of the RVLM through the release of GABA [12]. Barosensitive neurons in the RVLM accept the inhibitory information and then project to sympathetic preganglionic neurons in the thoracic spinal cord. This eventually reduces the sympathetic activity [4]. The baroreflex may also involve another regulatory region in this complicated circuit. More studies are needed to find out all of its mechanisms.

The afferent impulses activated by arterial BP pass by the NTS, CVLM and RVLM, reach sympathetic preganglionic neurons leading to a decrease in sympathetic activity as well as an increase in parasympathetic activity, and complete the feedback circuit of the carotid baroreflex. This results in bradycardia, decreased cardiac contractility and decreased peripheral vascular resistance [13]. These baroreflex mechanisms play a significant role in the shortterm regulation of $\mathrm{BP}[14]$.

\section{Baroreflex Sensitivity}

Baroreflex sensitivity (BRS) represents the performance of the carotid baroreflex, which can be quantified by the heart rate response to changes in BP. Several 'classic' methods have been developed to examine BRS, such as the pharmacologic way, the Valsalva maneuver and the neck chamber technique.

Angiotensin was once used as a pressor to increase BP; then, subsequently, decreasing heart rate was observed [15]. However, angiotensin has been replaced by the $\alpha$-receptorstimulating drug phenylephrine. Phenylephrine (50-200 $\mu \mathrm{g}$ ) is administered until the systolic 
BP has increased by $>15$ and $<40 \mathrm{~mm} \mathrm{Hg}$. A consecutive and simultaneous recording of ECG and beat-to-beat arterial pressure is performed after an intravenous bolus of phenylephrine. The relationship between systolic BP and RR interval changes is fit for linear regression. The correlation coefficient represents the BRS; the lower the correlation coefficient the lower the BRS [16]. Three or more administrations of phenylephrine are performed at 5- to 10-min intervals, and the mean correlation coefficient is taken as a measurement of the BRS. However, the drug may change the transduction properties of baroreceptors and the response of the carotid sinus [17].

The Valsalva maneuver affects the blood circulation and autonomic function through closing the glottis and exhaling forcefully, voluntarily increasing the intrathoracic and abdominal pressure. The whole process is divided into four periods. Phase 4 is mostly used to quantify BRS [18]. The maneuver is generally done in the supine position with recording of ECG and beat-to-beat arterial pressure, which is repeated three times at 5-min intervals. A linear regression analysis is performed between the systolic BP and RR interval changes during the whole phase 4 or the overshoot part to analyze the BRS. However, the application of the Valsalva maneuver seems to be limited in patients with advanced heart disease [19].

Another important technique to measure BRS is the application of variable pressure to the neck by means of a sealed chamber, thus altering carotid sinus transmural pressure [20]. A positive pressure in the neck chamber simulates a decrease in arterial pressure and elicits the deactivation of CSBs, resulting in an increase in BP. The recorded connection between systolic BP and RR interval changes is also fit for linear regression. The neck chamber is an effective method to measure BRS. However, transmission of external pressure to the carotid sinus is imperfect [20]. It is mostly used in laboratory studies for pathophysiological investigations [21].

In addition, the analysis of spontaneous beat-to-beat fluctuations in BP and RR interval by computer-based techniques seems to have more advantages over other methods [22]. This includes the sequence method and spectral method. It has a better reproducibility because of its automatic and standardized computations [23, 24]. The sequence method records three or more consecutive beats and then analyzes the relationship between BP variability and related changes in RR interval through linear regression analysis. The spectral method contains two major power components, a high frequency and a low frequency $[25,26]$. The recorded systolic BP and RR interval signals are displayed and stored on a computer. The recorded data then go through a fast Fourier transformation and get a different frequency. It is proven that the mid-frequency band $(0.07-0.14 \mathrm{~Hz})$ can be an appropriate quantification of BRS [27]. This method may bring much less discomfort or risk to the patients. Due to its automatic and standardized computations, the results are not affected by the measuring method and can be applied repetitively [27].

The information of BRS is a significant predictor of clinical outcomes in cardiovascular diseases such as myocardial infarction or heart failure among others [22]. A 5-year follow-up study confirmed that an improvement in receptor sensitivity after carotid surgery was related to a long-lasting reduction in BP levels and variability. Baroreceptor function may be considered as an indirect indicator of both short-term and long-term efficacy [28].

\section{Hemodynamic Changes Associated with CEA and CAS}

CEA has become an important approach to the treatment of carotid stenosis and occlusion by removing atheroma from the carotid bifurcation and internal carotid artery. The surgery may decrease the subsequent risk of fatal or disabling stroke in patients with significant carotid stenosis. However, hemodynamic instability, especially postoperative hypertension, 
is very common after CEA $[29,30]$, which can directly or indirectly influence morbidity and mortality after the surgery. It is also associated with increasing use of critical care facilities and longer hospitalization [31]. A possible explanation for this phenomenon is that CEA would damage the carotid sinus and then impair BP homeostasis. In addition, other important factors also affect the hemodynamic instability, such as baroreceptor function, surgical factors and anesthetic factors.

The hemodynamic instability caused by CEA has a close relationship with the baroreceptor function. Baroreflex plays an important role in regulating arterial BP and is altered in different disease states, such as chronic hypertension [32], diabetes, recent transient ischemic attack or stroke [33], contralateral carotid stenosis and so on. Research has shown that patients with contralateral carotid stenosis are more likely to present baroreflex dysfunction and hemodynamic instability as well as a hypertensive shift after CEA [34]. These comorbidities are prevalent in patients presenting for CEA. Surgical removal of the carotid plaque causes immediate partial disruption of baroreceptor activity, leading to hypertension and increased arterial pressure instability.

Different surgical methods result in different types of hemodynamic instability. CEA contains two surgical methods which are frequently used, standard CEA (s-CEA) and eversion CEA (e-CEA). s-CEA is performed through a longitudinal arteriotomy on the anterior surface of the common carotid artery and internal carotid artery, which is closed with or without a patch. e-CEA is performed through a transverse incision of the internal carotid artery at the base of the carotid bulb. It is associated with more postoperative hypertension and a greater use of vasodilators after the operation than s-CEA [29]. The procedure itself may be responsible for the increased postoperative hypertension in e-CEA patients [29]. It is inevitable to transect the longitudinal carotid sinus nerve fibers located in the adventitia during the e-CEA. The destruction of baroreceptors results in the activation of the sympathetic nervous system and hypertension.

There is no significant difference in neurologic mortality when using either general (GA) or regional anesthesia (RA) [35]. However, the choice of the anesthetic method for CEA also affects the postoperative hemodynamic profile. CEA performed with RA leads to significantly less postoperative hemodynamic instability than CEA performed with GA, and these patients receive less vasoactive medications during surgery and have a shorter duration of hospitalization [35-37]. However, the specific mechanism is unclear. Maybe the change of baroreceptor function caused by the manipulation is abolished by infiltration of the local anesthetic into the periadventitial tissue around the carotid sinus [38]. Different GA drugs may have different effects on the cardiovascular function as well as baroreflex activity, resulting in differences in arterial pressure when using either RA or GA. This may give guidance for the patients with substantial cardiac risks who would poorly tolerate significant BP and heart rate shifts.

Postoperative hypertension caused by CEA is associated with stroke, death and, possibly, cardiac complications [1]. It is essential to control perioperative BP, but there is no consensus on how to determine the reference value of BP. Relevant literature points out the data in a reliable way [39]. Preoperative BP should be maintained in a proper range to avoid excessive decreases in cerebral perfusion. It seems reasonable to aim for a systemic arterial pressure $<180 \mathrm{~mm}$ Hg. Alpha- and beta-blockers may be more favorable. During the surgery, it is better to maintain the BP between the normal and $20 \%$ above baseline level. This can avoid 'watershed' stroke during the period of carotid cross-clamping. After the surgery, target systolic BP $<160 \mathrm{~mm} \mathrm{Hg}$ is widely used, but a lower threshold may be appropriate in those at a high risk for cerebral hyperperfusion or wound hematoma. Alpha- and beta-blockers are effective for the prevention or treatment of postoperative hypertension. In short, monitoring of BP during the perioperative period is the most important thing. 
CAS emerged as a less invasive alternative interventional therapy for carotid stenosis, especially for patients at a high surgical risk and those younger than 70 years [40]. However, hemodynamic depression including arterial hypotension and bradycardia often occurs during and after CAS [41]. The frequency of hemodynamic depression is reported to be between 13 and $75.9 \%$ [42-46]. Different definitions of hemodynamic depression, the procedural variables and the relatively small study population may account for the wide variation in hemodynamic depression frequency. This phenomenon may be due to baroreceptor dysfunction caused by the manipulation of the carotid sinus [47]. During the procedure of CAS, the CSBs are stretched by the angioplasty balloon and stent, which is similar to the stimulation of BP. CSBs sense the stretching and send impulses to the brainstem. Subsequently, a decline in BP and heart rate is observed. Risk factors associated with hemodynamic depression include increased age, decreased ejection fraction, coronary artery disease, balloon-expandable stents, bifurcation location of carotid stenosis, stenosis $>70 \%$, presence of contralateral stenosis, severely calcified plaque and the plaque length $[2,42,44-46]$. However, patients with postendarterectomy stenosis have a lower frequency of hemodynamic depression [41]. Severe hemodynamic depression may decrease the perfusion of the brain, potentially leading to neurologic and cardiac complications such as transient ischemic attack, minor stroke or major stroke. It may also increase short-term complications and long-term mortality [2], especially in patients with persistent hypotension [41]. Careful medication or other preventive methods are necessary to prevent hemodynamic depression.

Diehm et al. [48] conducted a study on the influence of stent type on hemodynamic depression after CAS and drew the conclusion that nitinol stents were associated with a higher risk of hypotension compared with braided Elgiloy stents during the procedure. However, procedural hypotension and bradycardia were not related to the incidence of major adverse events but were associated with increased hospitalization time. This conclusion was also confirmed by Nii et al. [49]. In addition, Setacci et al. [50] proved that cutting balloon angioplasty using low expansion pressure was an effective method to prevent hemodynamic depression in patients with severe carotid artery calcification. Cutting balloon angioplasty may not only lead to important luminal gain but also avoid the elastic recoil of the lesion. Furthermore, transcutaneous temporary cardiac pacing, as an available preventive method, has been proven to be effective against angioplasty-induced bradycardia and hypotension [51].

Drug therapies of postoperative hemodynamic depression are very common. Atropine is usually used to treat postoperative hemodynamic depression. The administration of prophylactic atropine before balloon inflation during the procedure of CAS decreases the incidence of intraoperative bradycardia and hypotension [52]. However, the use of atropine has side effects such as dysrhythmia and tachycardia. Preventive application of glycopyrrolate acting on acetylcholine receptors can decrease postoperative bradycardia, hypotension, and tachycardia and is superior to atropine [53].

Further investigations are required to determine effective means which can blunt the hemodynamic alterations occurring during CAS.

\section{BRS Associated with CEA and CAS}

BRS index is a significant and reliable clinical predictor of cardiovascular morbidity and mortality [54]. A decreased BRS index has been shown to be related to increased sympathetic activity, which can lead to increased coronary vasoconstriction, platelet aggregation, impaired ventricular remodeling, and even to life-threatening arrhythmia [55, 56]. BRS is not only affected in patients with various kinds of physical diseases, but also in patients undergoing carotid surgery. The changes of BRS after CEA and CAS have been studied for a long time. 
Reduced carotid sinus sensitivity caused by CEA has been confirmed by many studies [34, $57,58]$. Excision of extensive intima and media leads to stiffness of the vessel wall, which may be responsible for this result [59]. It may also result from damage to CSBs or carotid sinus nerve during the surgery. However, the opposite has also been confirmed [60]. The removal of thickened intima and a part of media during CEA makes the arterial wall thinner and weaker. It can result in more expansion at the same pressure as before, leading to increased baroreceptor stimulation as well as increased BRS [59]. Patients with contralateral carotid stenosis showed greater baroreflex dysfunction by reducing the physiological baroreflex reserve [34]. Surgery methods also affect the carotid sinus sensitivity. There is a decrease in BRS after e-CEA and an increase after s-CEA [57].

Research of the relationship between BRS and CAS also shows different results. Both short-term changes and long-term effects in autonomic activity after CAS are observed. Increased parasympathetic modulation is obvious after CAS, which is likely mediated by the increased sensitivity of CSBs [61]. The parasympathetic predominance postoperatively was also confirmed by Yakhou et al. [62] and Alici et al. [63]. All of them recorded the short-term period after the surgery. Several reasons may be responsible for the increased BRS values. First of all, the surgery need not have an incision, which can reduce the injury to the baroreceptors and nerve fibers compared with the CEA. In addition, the stent exerts a continuous stress to the carotid vessel wall, which may increase the afferent impulses more or less. Another study that focused on the long-term $(1,053 \pm 833$ days $)$ effects of CAS on baroreflex function observed a significant reduction in BRS which was mainly due to the underlying disease rather than the CAS procedure [64]. This implies that the CAS surgery has no influence on the BRS values. Currently, it has been confirmed that the BRS decreases significantly 1 month after stenting and returns to baseline levels 6 months after CAS [65]. The vessels with a stent are more rigid than before and it takes time to adjust to this through the compensatory effect of contralateral baroreflex or other mechanisms which may account for the observed phenomenon. Furthermore, the BRS values can also be affected by case series, definition of BRS boundaries, and the measuring method. Thus, there might be inconsistent results. Since the change of BRS is not permanent, short-term monitoring is necessary.

BRS is an indirect indicator of postoperative recovery [28] and it represents the sensitivity of the carotid sinus. The variation of BRS induces hemodynamic instability. This is detrimental to postoperative recovery and long-term prognosis. We should try to avoid this kind of phenomenon in the clinical work. Careful monitoring is necessary especially in patients with severe underlying diseases which can blunt the BRS.

\section{Conclusion}

Hemodynamic changes are closely related to BRS variation. Severe hypertension occurs more frequently after CEA, while hemodynamic depression including perioperative hypotension or bradycardia occurs more frequently after CAS. Since hemodynamic instability is related to the patients' postoperative recovery, it is necessary to prevent or treat these conditions early. There are controversial opinions on the changes of BRS caused by CEA and CAS. However, there is no evident difference in short-term [66] and long-term [67] outcomes of the two main interventional ways to treat carotid artery stenosis. CAS is an alternative method to CEA, especially for patients at a high surgical risk and those younger than 70 years [40]. Here we discussed the hemodynamic instability and the variation of BRS. The results may provide useful information for the choice between the two interventional methods. However, these conclusions still need more experiments to confirm the effects. Most importantly, we should pay more attention to the physiological index of BRS in the future. 
Cao et al.: Hemodynamic Changes and Baroreflex Sensitivity Associated with Carotid Endarterectomy and Carotid Artery Stenting

\section{References}

1 Wong JH, Findlay JM, Suarez-Almazor ME: Hemodynamic instability after carotid endarterectomy: risk factors and associations with operative complications. Neurosurgery 1997;41:35-43.

-2 Dangas G, et al: Postprocedural hypotension after carotid artery stent placement: predictors and short- and long-term clinical outcomes. Radiology 2000;215:677-683.

3 Schreihofer AM, Guyenet PG: The baroreflex and beyond: control of sympathetic vasomotor tone by GABAergic neurons in the ventrolateral medulla. Clin Exp Pharmacol Physiol 2002;29:514-521.

4 Aicher SA, et al: Anatomical substrates for baroreflex sympathoinhibition in the rat. Brain Res Bull 2000;51: 107-110.

5 Palkovits M: Neuropeptides and biogenic amines in central cardiovascular control mechanisms; in Buckley JP, Ferario CM (eds): Central Nervous System Mechanisms in Hypertension. New York, Raven Press, 1981, pp 73-87.

-6 Andersen MC, Kunze DL: Nucleus tractus solitarius - gateway to neural circulatory control. Annu Rev Physiol 1994;56:93-116.

7 Seagard J, et al: Selective contribution of two types of carotid sinus baroreceptors to the control of blood pressure. Circ Res 1993;72:1011-1022.

8 Talman WT, Perrone MH, Reis DJ: Evidence for L-glutamate as the neurotransmitter of baroreceptor afferent nerve fibers. Science 1980;209:813-815.

-9 Jeske I, et al: Identification of baroreceptor reflex interneurons in the caudal ventrolateral medulla. Am J Physiol 1993;264:R169-R178.

$\checkmark 10$ Aicher SA, et al: Nucleus tractus solitarius efferent terminals synapse on neurons in the caudal ventrolateral medulla that project to the rostral ventrolateral medulla. Brain Res 1995;693:51-63.

-11 Chan RK, Sawchenko PE: Organization and transmitter specificity of medullary neurons activated by sustained hypertension: implications for understanding baroreceptor reflex circuitry. J Neurosci 1998;18: 371-387.

12 Jeske I, Reis D, Milner T: Neurons in the barosensory area of the caudal ventrolateral medulla project monosynaptically on to sympathoexcitatory bulbospinal neurons in the rostral ventrolateral medulla. Neuroscience 1995;65:343-353.

13 Kirchheim HR: Systemic arterial baroreceptor reflexes. Physiol Rev 1976;56:100-177.

14 Mancia G, et al: Control of blood pressure by carotid sinus baroreceptors in human beings. Am J Cardiol 1979; 44:895-902.

15 Smyth HS, Sleight P, Pickering GW: Reflex regulation of arterial pressure during sleep in man. A quantitative method of assessing baroreflex sensitivity. Circ Res 1969;24:109-121.

16 Gribbin B, et al: Effect of age and high blood pressure on barorefiex sensitivity in man. Circ Res 1971;29:424431.

17 Eckberg DL, Sleight P: Human Baroreflexes in Health and Disease. Oxford, Clarendon Press, 1992.

18 Palmero H, et al: Baroreceptor reflex sensitivity index derived from phase 4 of the Valsalva maneuver. Hypertension 1981;3:II-134.

$>19$ Rovere L: Assessment of baroreflex sensitivity in patients with preserved and impaired left ventricular function by means of the Valsalva manoeuvre and the phenylephrine test. Clin Sci 2001;100:33-41.

20 Ludbrook J, et al: The variable-pressure neck-chamber method for studying the carotid baroreflex in man. Clin Sci Mol Med 1977;53:165-171.

21 Sleight P, et al: Physiology and pathophysiology of heart rate and blood pressure variability in humans: is power spectral analysis largely an index of baroreflex gain? Clin Sci 1995;88:103-109.

22 La Rovere MT, Pinna GD, Raczak G: Baroreflex sensitivity: measurement and clinical implications. Ann Noninvasive Electrocardiol 2008;13:191-207.

23 Legramante M, et al: Evaluation of reproducibility of spontaneous baroreflex sensitivity at rest and during laboratory tests. J Hypertens 1996;14:1099-1104.

24 Parlow J, et al: Spontaneous cardiac baroreflex in humans. Comparison with drug-induced responses. Hypertension 1995;25:1058-1068.

25 Pagani M, et al: Power spectral analysis of heart rate and arterial pressure variabilities as a marker of sympatho-vagal interaction in man and conscious dog. Circ Res 1986;59:178-193.

-26 Malliani A, et al: Cardiovascular neural regulation explored in the frequency domain. Circulation 1991;84: 482-492.

27 Robbe HW, et al: Assessment of baroreceptor reflex sensitivity by means of spectral analysis. Hypertension 1987; 10:538-543.

28 Hirschl M, Kundi M, Blazek G: Five-year follow-up of patients after thromboendarterectomy of the internal carotid artery relevance of baroreceptor sensitivity. Stroke 1996;27:1167-1172.

29 Mehta M, et al: Eversion technique increases the risk for post-carotid endarterectomy hypertension. J Vasc Surg 2001;34:839-845.

30 van Mook WNKA, et al: Cerebral hyperperfusion syndrome. Lancet Neurol 2005;4:877-888.

-31 Hartsell PA, et al: Postoperative blood pressure changes associated with cervical block versus general anesthesia following carotid endarterectomy. Ann Vasc Surg 1999;13:104-108. 
Cao et al.: Hemodynamic Changes and Baroreflex Sensitivity Associated with Carotid

32 Comfere T, et al: Angiotensin system inhibitors in a general surgical population. Anesth Analg 2005;100:636644.

-33 Eames P, et al: Dynamic cerebral autoregulation and beat to beat blood pressure control are impaired in acute ischaemic stroke. J Neurol Neurosurg Psychiatry 2002;72:467-472.

-34 Nouraei SA, et al: Carotid endarterectomy impairs blood pressure homeostasis by reducing the physiologic baroreflex reserve. J Vasc Surg 2005;41:631-637.

35 Sternbach Y, et al: Hemodynamic benefits of regional anesthesia for carotid endarterectomy. J Vasc Surg 2002; 35:333-339.

36 Bandić Pavlović D, et al: The hemodynamic effect of intermediate cervical plexus block compared to general anesthesia in high risk patients with carotid endarterectomy. Period Biol 2013;115:295-297.

-37 Eibes TA, Gross WS: The influence of anesthetic technique on perioperative blood pressure control after carotid endarterectomy. Am Surg 2000;66:641-647.

-38 Al-Rawi PG, Sigaudo-Roussel D, Gaunt ME: Effect of lignocaine injection in carotid sinus on baroreceptor sensitivity during carotid endarterectomy. J Vasc Surg 2004;39:1288-1294.

-39 Stoneham M, Thompson J: Arterial pressure management and carotid endarterectomy. Br J Anaesth 2009;102: 442-452.

40 Roffi M, Mukherjee D, Clair DG: Carotid artery stenting vs endarterectomy. Eur Heart J 2009;30:26932704.

-41 Lin PH, et al: Factors associated with hypotension and bradycardia after carotid angioplasty and stenting. J Vasc Surg 2007;46:846-853; discussion 853-854.

42 Cieri E, et al: Is haemodynamic depression during carotid stenting a predictor of peri-procedural complications? Eur J Vasc Endovasc Surg 2008;35:399-404.

43 Leisch F, et al: Carotid sinus reactions during carotid artery stenting: predictors, incidence, and influence on clinical outcome. Catheter Cardiovasc Interv 2003;58:516-523.

44 Qureshi AI, et al: Frequency and determinants of postprocedural hemodynamic instability after carotid angioplasty and stenting. Stroke 1999;30:2086-2093.

45 Mlekusch W, et al: Hypotension and bradycardia after elective carotid stenting: frequency and risk factors. J Endovasc Ther 2003;10:851-859; discussion 860-861.

46 Lian X, et al: Risk factors associated with haemodynamic depression during and after carotid artery stenting. J Clin Neurosci 2011;18:1325-1328.

47 Gupta R, et al: Rate, predictors, and consequences of hemodynamic depression after carotid artery stenting. I Am Coll Cardiol 2006;47:1538-1543.

48 Diehm N, et al: Influence of stent type on hemodynamic depression after carotid artery stent placement. J Vasc Interv Radiol 2008;19:23-30.

49 Nii K, et al: Incidence of hemodynamic depression after carotid artery stenting using different self-expandable stent types. Neurol Med Chir 2011;51:556-560.

50 Setacci F, et al: Carotid highly-calcified de novo stenosis and cutting-balloon angioplasty: a tool to prevent haemodynamic depression? J Cardiovasc Surg 2009;50:357-364.

51 Im S-H, et al: Transcutaneous temporary cardiac pacing in carotid stenting: noninvasive prevention of angioplasty-induced bradycardia and hypotension. J Endovasc Ther 2008;15:110-116.

52 Cayne NS, et al: Carotid angioplasty and stent-induced bradycardia and hypotension: impact of prophylactic atropine administration and prior carotid endarterectomy. J Vasc Surg 2005;41:956-961.

55 Chung C, et al: Improved hemodynamic outcomes with glycopyrrolate over atropine in carotid angioplasty and stenting. Perspect Vasc Surg Endovasc Ther 2010;22:164-170.

54 Lantelme P, et al: Spontaneous baroreflex sensitivity: toward an ideal index of cardiovascular risk in hypertension? J Hypertens 2002;20:935-944.

55 De Ferrari GM, et al: Baroreflex sensitivity, but not heart rate variability, is reduced in patients with life-threatening ventricular arrhythmias long after myocardial infarction. Am Heart J 1995;130:473-480.

56 Hohnloser SH, et al: Reflex versus tonic vagal activity as a prognostic parameter in patients with sustained ventricular tachycardia or ventricular fibrillation. Circulation 1994;89:1068-1073.

57 Demirel S, et al: Changes in baroreceptor sensitivity after eversion carotid endarterectomy. J Vasc Surg 2012; 55:1322-1328.

58 Sigaudo-Roussel D, et al: Deterioration in carotid baroreflex during carotid endarterectomy. J Vasc Surg 2002; 36:793-798.

59 Angell-James JE, Lumley J: The effects of carotid endarterectomy on the mechanical properties of the carotid sinus and carotid sinus nerve activity in atherosclerotic patients. Br J Surg 1974;61:805-810.

60 Landesberg G, et al: Step baroreflex response in awake patients undergoing carotid surgery: time- and frequency-domain analysis. Am J Physiol 1998;274:H1590-H1597.

61 Demirci M, et al: Carotid artery stenting and endarterectomy have different effects on heart rate variability. J Neurol Sci 2006;241:45-51.

62 Yakhou L, et al: Noninvasive investigation of autonomic activity after carotid stenting or carotid endarterectomy. J Vasc Surg 2006;44:472-479.

63 Alici G, et al: Evaluation of autonomic functions by heart rate variability after stenting in patients with carotid artery stenosis. Ann Noninvasive Electrocardiol 2013;18:126-129. 
Cao et al.: Hemodynamic Changes and Baroreflex Sensitivity Associated with Carotid Endarterectomy and Carotid Artery Stenting

64 Huang CC, et al: Long-term effects of baroreflex function after stenting in patients with carotid artery stenosis. Auton Neurosci 2010;158:100-104.

65 Hsu LC, et al: Cerebral hemodynamics and baroreflex sensitivity after carotid artery stenting. Acta Neurol Scand 2013;127:46-52.

66 Yadav JS, et al: Protected carotid-artery stenting versus endarterectomy in high-risk patients. N Engl J Med 2004;351:1493-1501.

67 Gurm HS, et al: Long-term results of carotid stenting versus endarterectomy in high-risk patients. N Engl J Med 2008;358:1572-1579. 